\title{
Presarcopenia and its Impact on Disability in Female Patients With Rheumatoid Arthritis
}

\author{
Meltem ALKAN MELİKOĞLU \\ Department of Physical Medicine and Rehabilitation, Division of Rheumatology, \\ Medical Faculty of Atatürk University, Erzurum, Turkey
}

\begin{abstract}
Objectives: This cross-sectional pilot study aims to investigate presarcopenia in female patients with rheumatoid arthritis (RA) and to evaluate its relationship to the disability assessment.

Patients and methods: Forty female patients with RA (mean age $48.3 \pm 8.3$; range 31 to 66 years) and 40 healthy controls (mean age $46.2 \pm 6.9$; range 31 to 58 years) matched for age, sex, and body mass index were included. Pain, morning stiffness duration, disease activity score, erythrocyte sedimentation rate, C-reactive protein, and Health Assessment Questionnaire (HAQ) were evaluated. Body compositions were assessed with whole body dual energy X-ray absorptiometry. The appendicular skeletal muscle mass and skeletal muscle mass index (SMI) of RA patients were compared to the controls and possible correlations between SMI, disease characteristics, and HAQ score were investigated.

Results: The body mass index values and percentages of obese, overweight, and healthy weight subjects were similar in the patient and control groups. However, appendicular skeletal muscle mass and SMI calculations were significantly lower, and the percentage of presarcopenia was significantly higher in patients with RA $(20 \%)$ than controls $(7 \%)(p<0.05)$. Although there was no significant correlation between SMI and other parameters, a significant negative correlation was determined between SMI and HAQ score in patients with RA ( $p<0.05)$.

Conclusion: We demonstrated lower SMI values and higher presarcopenia ratios in patients with RA than healthy controls. Independent from other disease characteristics, the inverse correlation between SMI and HAQ scores may contribute to understanding of the impact of the process on patient disability.

Keywords: Disability; presarcopenia; rheumatoid arthritis; sarcopenia.
\end{abstract}

Rheumatoid arthritis (RA) is a chronic inflammatory disorder that is associated with increased morbidity and mortality due to several metabolic deteriorations. The close association between chronic illnesses and metabolic deterioration has long been recognized. ${ }^{1}$ The spectrum of metabolic abnormalities secondary to chronic diseases such as RA is indeed wide. Decreased muscle mass is one of these conditions, and interest has been growing in this issue over the last few years. In regard to low muscle mass, sarcopenia and presarcopenia are two conditions related to each other. Sarcopenia is a syndrome characterized by progressive and generalized loss of skeletal muscle mass and strength with a risk of adverse outcomes such as physical disability, poor quality of life, and death. ${ }^{2}$ While sarcopenia diagnosis needs both low muscle mass and low muscle function criteria, presarcopenia was defined as low muscle mass only. ${ }^{3}$ Due to the lack of simple clinical, biochemical, or imaging measures, it is still difficult to establish a widely accepted definition of sarcopenia or presarcopenia that is suitable for use in research and clinical practice. Depending on the literature definition used today, even with a conservative estimate, the prevalence of sarcopenia in 60 to 70 year olds has been reported as 5 to $13 \% .{ }^{4}$ The impact of low muscle mass includes increased morbidity, disability, high costs of health care, and mortality. ${ }^{2}$ Although 
these conditions are primarily affecting the elderly, it is known that, like osteopenia, they can also develop in younger cases and are possibly related to certain conditions such as disuse, malnutrition, or inflammatory diseases. ${ }^{1}$ Presarcopenia characterized by low lean mass seems to be reasonable in RA since cytokinedriven hypermetabolism and reduced physical activity may both contribute to the loss of muscle mass. ${ }^{5}$ In these patients, presarcopenia may also be considered clinically important due to its potential additional impact on disability. Therefore, this cross-sectional pilot study aims to investigate presarcopenia in female patients with RA and to evaluate its relationship to the disability assessment.

\section{PATIENTS AND METHODS}

A total of 40 female patients with RA (mean age $48.3 \pm 8.3$; range 31 to 66 years) who met American College of Rheumatology 2010 classification criteria and 40 age- and sex-matched healthy controls (mean age 46.2 \pm 6.9 ; range 31 to 58 years) were included in this cross-sectional study, which was conducted between January 2013 to December 2013 at Department of Physical Medicine and Rehabilitation, Medical Faculty of Atatürk University. Since significant differences in adiposity between males and females have been observed in RA, only female patients with RA were included. ${ }^{6}$ We excluded patients with prosthetic or metallic elements, infections, anamnesis of trauma, recent surgery, cancer, stroke, pregnancy, or weight over $140 \mathrm{~kg}$. The study protocol was approved by the Medical Faculty of Atatürk University Ethics Committee. A written informed consent was obtained from each subject. The study was conducted in accordance with the principles of the Declaration of Helsinki.

Besides demographic data, patient pain evaluation, which was performed using a $10 \mathrm{~cm}$ visual analog scale, and duration of morning stiffness were recorded. RA disease activity was evaluated by using the 28-joint disease activity score (DAS28) (using erythrocyte sedimentation rate). ${ }^{7}$ Also, Health Assessment Questionnaire (HAQ), the most commonly used instrument for evaluating functional disability in RA and which was tested and validated for our population, was assessed. ${ }^{8,9}$ Body mass index (BMI) was calculated as body weight divided by the square of height $\left(\mathrm{kg} / \mathrm{m}^{2}\right)$. Erythrocyte sedimentation rate according to Westergren method and C-reactive protein concentration by nephelometry (Beckman Array Protein System, USA) were also recorded.

Body composition and regional fat and lean muscle mass distribution were assessed with whole body dual energy X-ray absorptiometry (DXA; Hologic QDR 2000, Hologic Inc., Bedford, USA). The DXA has been accepted as one of the most commonly used, widely available, and low cost technologies for measuring body composition and muscle mass estimation. It has been reported that it provides valid estimates of appendicular skeletal muscle mass, and skeletal muscle measures with DXA are associated with prevalent and incident physical disability. ${ }^{10}$ To define presarcopenia, it is necessary to have a measure of relative muscle mass. Various options to define subnormal values for muscle mass designation have been suggested. ${ }^{2}$ Baumgartner et al. ${ }^{11}$ calculated the muscle mass of the four limbs from a DXA scan as appendicular skeletal muscle mass (ASM) and defined a skeletal muscle mass index (SMI) as $\mathrm{ASM} /$ height $^{2}\left(\mathrm{~kg} / \mathrm{m}^{2}\right)$ since absolute muscle mass is correlated strongly with height. Since it has been reported that, when defined in this way, muscle mass was independent of ethnicity, age, comorbidity, and health behaviors, we also used this methodology in our study.

We compared the SMI of patients with RA to the controls. Cut off points for presarcopenia depended upon the measurement technique chosen and on the availability of reference studies. In our study, we used the same cut off points as the previous studies, which used young adults as a reference group. ${ }^{11,12}$ With this approach, the cut off values for low muscle mass (ASM/height ${ }^{2}$; $\mathrm{kg} / \mathrm{m}^{2}$ ) were defined as 5.45 for females. ${ }^{11}$ We also investigated possible correlations between SMI, disease characteristics, and HAQ.

\section{Statistical analysis}

All the statistical analyses were performed using the IBM SPSS version 20.0 software package program (IBM Corporation, Armonk, NY, USA). The data were analyzed for normality with Kolmogorov-Smirnov test. The independent samples t test, Chi-square test, and Pearson's 
Table 1. Demographic data, disease characteristics and body compositions of study participants

\begin{tabular}{|c|c|c|c|c|c|}
\hline & \multicolumn{2}{|c|}{ Patients with RA } & \multicolumn{2}{|c|}{ Healthy controls } & \multirow[b]{2}{*}{$p$} \\
\hline & $\%$ & Mean \pm SD & $\%$ & Mean \pm SD & \\
\hline Age (year) & & $48.3 \pm 8.3$ & & $46.2 \pm 6.9$ & NS \\
\hline Disease duration (months) & & $75.4 \pm 68.1$ & & - & \\
\hline Body mass index $\left(\mathrm{kg} / \mathrm{m}^{2}\right)$ & & $31.8 \pm 7.0$ & & $32.5 \pm 5.7$ & NS \\
\hline Obesity $(\mathrm{BMI} \geq 30)$ & 52 & & 66 & & NS \\
\hline Overweight $(25 \geq \mathrm{BMI}<29.5)$ & 34 & & 23 & & NS \\
\hline Healthy weight $(18.5 \geq \mathrm{BMI}<24.5)$ & 13 & & 10 & & NS \\
\hline Appendicular skeletal muscle mass (ASM) & & $15.2 \pm 3.5$ & & $17.4 \pm 2.4$ & 0.027 \\
\hline SMI (ASM/height $\left.{ }^{2}\right)\left(\mathrm{kg} / \mathrm{m}^{2}\right)$ & & $6.4 \pm 1.1$ & & $7.1 \pm 1.1$ & 0.014 \\
\hline Sarcopenia & 20 & & 7 & & 0.05 \\
\hline
\end{tabular}

correlation test were used to analyze the data. Statistical significance was determined as a $p$ value of $\leq 0.05$.

\section{RESULTS}

There was no significant difference in the BMI values of patients with RA and the healthy controls. The percentages of obese, overweight, and healthy weight subjects were also similar in the patient and control groups. However, ASM and SMI calculations were found to be significantly lower in patients with RA than in the controls $(p<0.05)$. When the cut off point was considered 5.45, as in the reference group of the previous study that we used, the methodology to define low muscle mass ${ }^{11}$ was determined in $20 \%$ of patients with RA and in 7\% of the controls. The percentage of presarcopenia was found to be significantly higher in patients with RA than in the controls $(\mathrm{p}<0.05)$. The demographic data and body compositions of the participants are shown in Table 1.

Although there were no significant correlations between SMI and age, disease duration, morning stiffness, pain, DAS28 levels, and laboratory investigations, a significant negative correlation was determined between SMI and HAQ score in patients with RA $(p<0.05)$. These correlations are shown in Table 2.

\section{DISCUSSION}

In this study, higher presarcopenia ratios were demonstrated in female patients with RA than healthy individuals. Independent from other disease characteristics, low muscle mass was found to be related to disability in the patients.

Besides the difficulty in defining low muscle mass in the general population, defining it seems to be even more difficult in patients with RA. Definition needs to depend on objective imaging techniques since it is difficult to directly relate the impairment of muscle function to low muscle mass in these patients. DXA is an attractive alternative method both for research and clinical use to distinguish fat, bone mineral, and lean tissues. DXA measurements have been validated against computed tomography and magnetic resonance imaging. ${ }^{11}$

On the other hand, using healthy young adults for cut off points for low muscle mass has been recommended. ${ }^{2}$ However, there is a lack of generally applicable young-normal values, making

Table 2. Correlations between disease characteristics and skeletal muscle mass index of patients with rheumatoid arthritis

\begin{tabular}{lcc}
\hline & \multicolumn{2}{c}{ SMI } \\
\cline { 2 - 3 } & Mean $\pm \mathrm{SD}$ & $p$ \\
\hline Age (year) & $48.3 \pm 8.3$ & $\mathrm{NS}$ \\
Disease duration (months) & $75.4 \pm 68.1$ & $\mathrm{NS}$ \\
Morning stiffness & $41.6 \pm 32.6$ & $\mathrm{NS}$ \\
Pain & $4.8 \pm 1.7$ & $\mathrm{NS}$ \\
Disease activity score 28 & $3.4 \pm 0.2$ & $\mathrm{NS}$ \\
Health assessment questionnaire & $0.7 \pm 0.1$ & 0,046 \\
Erythrocyte sedimentation rate & $22.2 \pm 5.1$ & $-0,238 \mathrm{r}$ \\
C-reactive protein & $0.41 \pm 0.1$ & $\mathrm{NS}$ \\
\hline SMI: Skeletal muscle mass index; SD: Standard deviation; NS: Not \\
significant.
\end{tabular}


it difficult to quantify the severity of skeletal muscle loss and estimate the prevalence of presarcopenia in healthy and diseased populations. ${ }^{13}$ In our study, we used the same cut off points as the previous study, which used young adults as recommended. ${ }^{11}$ We also compared the SMI of patients with RA to age, sex, and BMI similar healthy controls. In this way, we demonstrated that muscle mass was lower in patients with RA than their similar matches.

The prevalence of sarcopenia in RA has been reported as different rates in several studies, with one as high as $30 \% .^{5,6,14}$ In our study, we found that $20 \%$ of the patients with RA presented presarcopenia, and this rate was significantly higher than the controls. In spite of the difference among the rates in several studies that might be due to many factors, such as the methodology used in defining sarcopenia, ethnicity, and demographic features, previous studies agreed on risk of sarcopenia in patients with RA. Our study supports such findings with results showing significantly higher presarcopenia rates in RA patients versus controls.

The exact mechanisms causing low muscle mass in RA remain undetermined, but they seem to be multifactorial and include increased production of pro-inflammatory cytokines, mainly tumor necrosis factor-alpha and interleukin-1 beta, hormonal changes, oxidative damage, reduced protein synthesis in myocytes, insulin resistance, inadequate protein ingestion, and physical activity limitation. ${ }^{15}$ This state may give rise to a vicious cycle of decreased exercise, increased fatigue and weakness, and increased fat mass with implications for comorbidity and mortality. ${ }^{5}$

In our study, the majority of RA patients were overweight or obese, except for $13 \%$ of the patients with healthy body weight. However, muscle mass of the patients was significantly lower than the controls. It has been reported that sarcopenia in RA may be accompanied by maintenance or elevation of the fat mass, which results in limited or no weight loss. ${ }^{15}$ In conditions such as malignancy, RA, and ageing, lean body mass may decrease while fat mass may be preserved or may even increase. This state is called sarcopenic obesity, and thus the relationship between reductions of muscle mass is often independent of body mass. ${ }^{2}$ In previous studies, abnormalities in body composition (sarcopenia, overfat, and sarcopenic obesity) were reported to be greater in patients with $\mathrm{RA}$ than controls. ${ }^{16}$ The adipose tissue is known as a producer of several pro-inflammatory cytokines, such as tumor necrosis factor-alpha, interleukin-6, and interleukin-1, all of which are associated with aging, obesity, and low muscle mass. ${ }^{5}$

In our study, we could not demonstrate any significant relationship between muscle mass and disease characteristics, including disease duration, pain evaluation, morning stiffness duration, disease activity, and ages of the patients. In previous studies, there were different results regarding body composition and disease characteristics in RA. ${ }^{5,6,14}$ Although sarcopenia is primarily a disease of the elderly, it is known that decreased muscle mass may be associated with several conditions other than the aging process, and can also be seen in younger patients, such as those with inflammatory diseases. ${ }^{10}$ In the present study, the SMI of the patients with RA was found to be lower than the controls regardless of the ages of the participants. Thus, lower lean muscle mass may be associated with disease itself rather than age profile. Furthermore, disease duration was questioned as another possible related factor to muscle mass in previous studies. Giles et al. $^{16}$ reported a higher proportion of unhealthy body composition phenotype in older RA patients with a longer disease duration compared to controls. A significantly higher proportion of unhealthy body composition phenotypes, higher fat mass, and lower lean mass were also reported in female patients with early RA than the controls. ${ }^{5}$ Similar results were observed in another study on patients with early RA $\left(<1\right.$ year). ${ }^{17}$ Disease activity, erythrocyte sedimentation rate, and C-reactive protein levels also showed no correlation with SMI in our study. This may be due to the low or moderate disease activity score profile of the patients. However, Gamboa-Cardenas et al. ${ }^{18}$ reported a negative association between trunk to appendicular lean mass ratio and quality of life independent of age, socioeconomic status, rheumatoid factor, disease activity, joint damage, disease duration, and use of steroids in patients with RA. In another study, DAS28 score was positively correlated with fat mass and distribution, and 
these body composition changes were associated with seropositivity, HAQ, and DAS28 scores, but not with disease modifying antirheumatic drugs and glucocorticoid use which was supported by previous data. ${ }^{5,16}$ Higher C-reactive protein levels in sarcopenic patients with RA have also been reported. ${ }^{14}$ Although studies agreed on abnormal body composition in patients with RA, it seems that its proportion varies considerably and depends upon the degree of muscle mass reduction which is considered to be significant and the method used to estimate muscle mass. ${ }^{19}$

In our study, we found a significantly inverse correlation between SMI and HAQ scores of the RA patients. This result was in accordance with the previous studies investigating relationships between body composition and disability or muscle performance in patients with RA.5, ${ }^{5,6,14}$ Decreased lean mass has also been reported to be associated with decreased level of exercise and may lead to increased insulin resistance and cardiovascular comorbidity in RA patients. ${ }^{20}$ Besides the inflammatory nature of the disease, it is also possible that disability could lead to low muscle mass by limiting physical activity, or decreased muscle mass in these patients may subsequently predispose them to impaired ability to perform daily activities. Independent from the cause, reduced lean body mass seems to have an impact on disability in patients with RA.

Contributing to these findings, similar results have been shown in the other patient groups. In a previous study, low muscle mass was found to be associated with functional impairment and disability independent of chronic morbidity, which is a potential risk factor for functional impairment. ${ }^{21}$ In studies on the elderly, low muscle mass represented an impaired state of health with high personal mobility disorders, increased risk of falls and fractures, impaired ability to perform activities of daily living, disabilities, loss of independence, and increased risk of death. ${ }^{2,22-25}$ Low muscle mass was reported to be associated with a three to fourfold likelihood of disability in elderly people, independent of age, sex, obesity, ethnicity, socioeconomic status, chronic morbidity, and health behaviors. ${ }^{11}$

Based on increased awareness of muscle mass reduction and its consequences in the general population, prevention methods, such as dietary strategies, lifestyle changes, and exercise regimens, have come into question in the treatment. However, no standardized therapy aimed specifically at that aspect of RA has been proposed, and the effects of the current treatments have not been well studied despite the potential socioeconomic and functional impact on that population. ${ }^{15}$

Despite the progress in understanding the molecular mechanisms that lead to muscle atrophy in several situations, rheumatoid sarcopenia/ presarcopenia is still rarely studied. Further studies are needed since better understanding of the mechanisms of modulation between muscle catabolism and anabolism may result in the development of innovative and more effective therapeutic strategies and better quality of life for the patients.

There were limitations in our pilot study investigating presarcopenia in RA. First of all, the definition of low presarcopenia needed to be based on the general definition due to the lack of a specific definition for these patients. Since there is no existing research about the cut off points for presarcopenia in our population, we used the methodology that was reported to be independent from ethnicity. The other limitation was the lack of evaluation of any relationship between drug usage and low muscle mass. Since the disease duration was relatively long in our study group, there was an extremely heterogenic previous drug usage anamnesis. Future studies on homogenous treatment groups may cast light on the results.

By comparing patients with RA to age, sex, and BMI similar healthy controls, finding body composition differences from their matches and its relation to disability may increase the importance of our results. Although several studies have reported associations between muscle quality, mass, and functional impairments in the general population, future studies are needed to establish clinically relevant criteria to define those at relative risk of facing disability and morbidity in patients with RA.

In conclusion, in the present pilot study, we demonstrated higher presarcopenia ratios in patients with RA compared to their age, sex, 
and BMI similar matches. Also, independent from other disease characteristics, the inverse correlation between SMI and HAQ scores found in our study may contribute to understanding the impact of the process on patient disability. These findings suggest that, in the management of the disease, clinicians should take into account the effect of body composition changes and their potential impact on disability in patients with RA.

\section{Declaration of conflicting interests}

The author declared no conflicts of interest with respect to the authorship and/or publication of this article.

\section{Funding}

The author received no financial support for the research and/or authorship of this article.

\section{REFERENCES}

1. Muscaritoli M, Anker SD, Argilés J, Aversa Z, Bauer $\mathrm{JM}$, Biolo G, et al. Consensus definition of sarcopenia, cachexia and pre-cachexia: joint document elaborated by Special Interest Groups (SIG) "cachexia-anorexia in chronic wasting diseases" and "nutrition in geriatrics". Clin Nutr 2010;29:154-9.

2. Cruz-Jentoft AJ, Baeyens JP, Bauer JM, Boirie Y, Cederholm T, Landi F, et al. Sarcopenia: European consensus on definition and diagnosis: Report of the European Working Group on Sarcopenia in Older People. Age Ageing 2010;39:412-23.

3. Han DS, Chang KV, Li CM, Lin YH, Kao TW, Tsai $\mathrm{KS}$, et al. Skeletal muscle mass adjusted by height correlated better with muscular functions than that adjusted by body weight in defining sarcopenia. Sci Rep 2016;6:19457.

4. Phillips SM. Nutritional supplements in support of resistance exercise to counter age-related sarcopenia. Adv Nutr 2015;6:452-60.

5. Dao HH, Do QT, Sakamoto J. Abnormal body composition phenotypes in Vietnamese women with early rheumatoid arthritis. Rheumatology (Oxford) 2011;50:1250-8.

6. Baker JF, Long J, Ibrahim S, Leonard MB, Katz P. Are men at greater risk of lean mass deficits in rheumatoid arthritis? Arthritis Care Res (Hoboken) 2015;67:112-9.

7. Prevoo ML, van 't Hof MA, Kuper HH, van Leeuwen MA, van de Putte LB, van Riel PL. Modified disease activity scores that include twenty-eight-joint counts. Development and validation in a prospective longitudinal study of patients with rheumatoid arthritis. Arthritis Rheum 1995;38:44-8.
8. Wolfe F, Kleinheksel SM, Cathey MA, Hawley DJ, Spitz PW, Fries JF. The clinical value of the Stanford Health Assessment Questionnaire Functional Disability Index in patients with rheumatoid arthritis. J Rheumatol 1988;15:1480-8.

9. Küçükdeveci AA, Sahin H, Ataman S, Griffiths B, Tennant A. Issues in cross-cultural validity: example from the adaptation, reliability, and validity testing of a Turkish version of the Stanford Health Assessment Questionnaire. Arthritis Rheum 2004;51:14-9.

10. Pahor M, Manini T, Cesari M. Sarcopenia: clinical evaluation, biological markers and other evaluation tools. J Nutr Health Aging 2009;13:724-8.

11. Baumgartner RN, Koehler KM, Gallagher D, Romero L, Heymsfield SB, Ross RR, et al. Epidemiology of sarcopenia among the elderly in New Mexico. Am J Epidemiol 1998;147:755-63.

12. Gallagher D, Visser M, De Meersman RE, Sepúlveda D, Baumgartner RN, Pierson RN, et al. Appendicular skeletal muscle mass: effects of age, gender, and ethnicity. J Appl Physiol (1985) 1997;83:229-39.

13. Tankó LB, Movsesyan L, Mouritzen U, Christiansen C, Svendsen OL. Appendicular lean tissue mass and the prevalence of sarcopenia among healthy women. Metabolism 2002;51:69-74.

14. Ferraz-Amaro I, Delgado-Frias E, HernandezHernandez V, Muniz JR, Gonzalez-Diaz A, RodriguezBethencourt AG, et al. Abdominal Adiposity and Body Composition in Rheumatoid Arthritis: realtion with disease characteristics in a case control study. Arthritis Rheum 2013;65:577-8.

15. Teixeira Vde O, Filippin LI, Xavier RM. Mechanisms of muscle wasting in sarcopenia. Rev Bras Reumatol 2012;52:252-9.

16. Giles JT, Ling SM, Ferrucci L, Bartlett SJ, Andersen $\mathrm{RE}$, Towns $\mathrm{M}$, et al. Abnormal body composition phenotypes in older rheumatoid arthritis patients: association with disease characteristics and pharmacotherapies. Arthritis Rheum 2008;59:807-15.

17. Book C, Karlsson MK, Akesson K, Jacobsson LT. Early rheumatoid arthritis and body composition. Rheumatology (Oxford) 2009;48:1128-32.

18. Gamboa-Cardenas RV, Ugarte-Gil MF, Noriega E, Medina-Chinchon M, Zevallos-Miranda F, CuchoVenegas JM, et al. Lean body mass (LBM) distribution negatively impacts on health related quality of life (HRQoL) in patients with rheumatoid arthritis (RA). Arthritis and Rheum 2013;65:181.

19. Summers GD, Deighton CM, Rennie MJ, Booth $\mathrm{AH}$. Rheumatoid cachexia: a clinical perspective. Rheumatology (Oxford) 2008;47(8):1124-31.

20. Bianchi G, Rossi V, Muscari A, Magalotti D, Zoli M. Physical activity is negatively associated with the metabolic syndrome in the elderly. QJM 2008;101:713-21.

21. Guralnik JM, Fried LP, Salive ME. Disability as a public health outcome in the aging population. Annu Rev Public Health 1996;17:25-46. 
22. Cawthon PM, Marshall LM, Michael Y, Dam TT, Ensrud KE, Barrett-Connor E, et al. Frailty in older men: prevalence, progression, and relationship with mortality. J Am Geriatr Soc 2007;55:1216-23.

23. Lauretani F, Russo CR, Bandinelli S, Bartali B, Cavazzini C, Di Iorio A, et al. Age-associated changes in skeletal muscles and their effect on mobility: an operational diagnosis of sarcopenia. J Appl Physiol
(1985) 2003;95:1851-60.

24. Rolland Y, Czerwinski S, Abellan Van Kan G, Morley JE, Cesari M, Onder G, et al. Sarcopenia: its assessment, etiology, pathogenesis, consequences and future perspectives. J Nutr Health Aging 2008;12:433-50.

25. Topinková E. Aging, disability and frailty. Ann Nutr Metab 2008;52 Suppl 1:6-11. 